\title{
Determinación de presiones críticas de la ventana de lodo, aplicando modelos analíticos y caracterizaciones geomecánicas*
}

\author{
Ingrid Natalia Muñoz Quijano ${ }^{a}$ Carlos Andrés Perdomo Lozada
}

\begin{abstract}
Resumen: en Colombia, las cuencas sedimentarias son estructuralmente complejas y presentan un alto tectonismo, por lo que la tendencia es que las operaciones de perforación en yacimientos de hidrocarburos sean complejas y, en consecuencia, causen pérdidas de tiempo y dinero a la industria de hidrocarburos. La aplicación de áreas del conocimiento como la geomecánica es una solución viable al desafío de estabilizar pozos. Este estudio propone algoritmos para evaluar los modelos preexistentes, con modelos nuevos que describen la concentración de los esfuerzos alrededor del pozo y el criterio de falla, estableciendo la relación de falla entre la resistencia de la roca y los esfuerzos que concentra. Ello permite describir el estado de esfuerzos de la pared del pozo, al combinar los criterios de fallas tensionales y compresionales, para obtener las presiones críticas de fractura y colapso, respectivamente. Junto con la presión de poro, aquellas presiones son los componentes de la ventana de lodo, con la que puede mejorarse la estabilidad del pozo, a partir del peso del lodo y la dirección del pozo. Se encontró que, en modelos tensionales, con respecto a la presión crítica tensional, las regiones se encuentran para $A z_{\text {pozo }}$ entre $150-330^{\circ}$ y $180-360^{\circ}$ e Inc $c_{\text {pozo }}$ entre $60^{\circ}$ y $90^{\circ}$; también, que valores menores del overbalanced concuerdan con la dirección más estable, que se encuentra para Inc $_{\text {pozo }}$ en $0-20^{\circ}$, aproximadamente, lo que hace que los pozos de poca inclinación tengan distribución más uniforme de esfuerzos, en comparación con los pozos altamente desviados.
\end{abstract}

Palabras clave: criterio de falla; estabilidad de pozos; geomecánica; presión crítica de colapso; presión crítica de fractura; ventana de lodo.

Recibido: 16/04/2020 Aceptado: 12/04/2021 Disponible en línea: 23/07/2021

* Artículo de investigación.

a Doctora en Geología de la Universidad de Heidelberg, profesora asociada de Ingeniería de Petróleos, Universidad Surcolombiana, Neiva, Colombia. Correo electrónico: ingrid.munoz@usco.edu.co ORCID: https:// orcid.org/0000-0002-3518-0182

b Ingeniero de Petróleos, Universidad Surcolombiana, Neiva, Colombia. Correo electrónico: carlos.950301@ gmail.com. ORCID: https://orcid.org/0000-0002-7997-9180 
Cómo citar: I. N. Muñoz Quijano y C. A. Perdomo Lozada, «Determinación de presiones críticas de la ventana de lodo, aplicando modelos analíticos y caracterizaciones geomecánicas», Cien.Ing. Neogranadina, vol. 31, n. ${ }^{\circ}$, pp. 25-36, jul. 2021.

\title{
Determination of Critical Mud Window Pressures by Applying Analytical Models and Geo-Mechanical Characterization
}

\begin{abstract}
In Colombia, sedimentary basins are structurally complex and have a high tectonism, meaning that drilling operations in hydrocarbon deposits are also complex and, consequently, causing time and money losses to the hydrocarbons industry. Applying areas of knowledge such as geomechanics is a viable solution to the challenge of stabilizing wells. This study proposes algorithms to evaluate pre-existing models, with new models describing the concentration of the stresses around the well and the failure criterion, establishing the failure relationship between rock resistance and the stresses it concentrates. This allows to describe the stress state of the well wall by combining tension and compressional failure criteria to obtain critical fracture and collapse pressures, respectively. Along with pore pressure, those pressures are the components of the mud window, with which the well stability can be improved, from the weight of the mud and the direction of the well. It was found that, in tension models, with respect to tension critical pressure, the regions are found for $A z_{\text {well }}$ between $150-330^{\circ}$ and $180-360^{\circ}$ and $I_{n c_{w e l l}}$ between $60^{\circ}$ and $90^{\circ}$. Also, that lower overbalanced values agree with the most stable direction, which is found for $\mathrm{Inc}_{\text {well }}$ at approximately $0-20^{\circ}$, which makes low-slope wells to show more evenly distributed stress, compared to highly diverted wells.
\end{abstract}

Keywords: Failure criterion, well stability, geomechanics, critical collapse pressure, critical fracture pressure, mud window. 


\section{Introducción}

La explotación de yacimientos representa retos a los ingenieros, debido a que cada vez es más común encontrar formaciones productoras con características geológicas y tectónicas de mayor complejidad. Estas formaciones deben ser estudiadas cuidadosamente, para determinar la manera más adecuada de extraer la mayor cantidad posible de petróleo y gas, con el fin de suplir la demanda energética y generar el incremento de las cifras de reservas y, en consecuencia, la producción de hidrocarburos.

En Colombia, la complejidad tectónica y estructural radica principalmente en la convergencia de las placas tectónicas Caribe, Nazca y Sudamericana. El movimiento del bloque norte de los Andes se estima hacia el noreste $\left(60^{\circ}\right)$ a una tasa de 8,6 mm/año en relación con la placa de América del Sur [1]. Por ello, las cuencas sedimentarias localizadas entre o cerca de la cordillera de los Andes (Occidental, Central y Oriental) están asociadas con regímenes de esfuerzos rumbo-deslizante o inverso. Esto significa que los esfuerzos horizontales son más grandes que los de sobrecarga, lo que, generalmente, ocasiona que las condiciones de perforación y completamiento de los pozos petroleros sean difíciles [2].

Una de las disciplinas que ha permitido una mejor comprensión y planeación de operaciones en zonas geológicamente complejas es la geomecánica, una ciencia de creciente aplicación a la industria petrolera [3]. Su desarrollo en yacimientos de petróleo y gas conduce a mejoras significativas en el desempeño económico [4], donde ha sido utilizada para diferentes caracterizaciones, que van desde el estudio de los factores en la fractura hidráulica [5], hasta modelamientos en campos de hidrocarburos en diferentes cuencas [6]-[8] e, incluso, investigaciones sobre mitigación de riesgos por sobrepresiones en pozo [9].

Con ello en mente, este estudio profundiza en la aplicación de la geomecánica en la estabilidad de pozos. Sin embargo, su rango de aplicabilidad va mucho más allá, y se involucra en la mayoría de los procesos de las fases de exploración, desarrollo y producción de hidrocarburos.

\section{Materiales y métodos}

Para el desarrollo de los modelos, se revisó la literatura y se tomaron planteamientos preexistentes, en lo que básicamente se combinaron dos aspectos: el primero describe la concentración de los esfuerzos alrededor del pozo; mientras que el segundo, denominado criterio de falla, estableció la relación de falla entre la resistencia de la roca y los esfuerzos que concentra, por lo que, si se superan los límites, la roca fallará y, como consecuencia, habrá inestabilidad en las paredes del pozo.

Luego, se propusieron los algoritmos para evaluar los modelos planteados. Teniendo en cuenta que los algoritmos incluyen muchos cálculos, se hizo necesario la programación de un código en Matlab $^{\oplus}$ que lo evaluara, para hacer las validaciones correspondientes.

Se trabajaron los modelos polares y cartesianos. En el primero, se ponen a variar todos los posibles valores de $\operatorname{Inc}_{\text {pozo }}\left(0-90^{\circ}\right)$ y de $\mathrm{Az}_{\text {pozo }}\left(0-360^{\circ}\right)$; mientras que en el segundo se especifica un valor de alguno de los dos y se pone a variar el otro.

\section{Estabilidad de pozos}

Antes de la perforación de un pozo petrolero, las rocas que conforman el yacimiento se encuentran en relativo equilibrio, sometidas solo a los esfuerzos in situ soportados por el macizo rocoso. Sin embargo, durante y después de la perforación, tal equilibrio se ve sometido a perturbaciones que, desde un punto de vista geométrico, pueden describirse como el cambio de un cilindro de roca por un cilindro de fluido (pozo con lodo), que ocasiona que los esfuerzos compresionales tiendan a concentrarse alrededor del hueco de la perforación [10]. Ello conduce a problemas de estabilidad en el pozo [11].

Para que exista estabilidad entre el hueco perforado y las rocas circundantes, debe predominar el equilibrio de todos los esfuerzos, es decir, que estos sean lo más parecidos posible. Para ello, deben controlarse la dirección del pozo y el peso del lodo [12]. La deficiencia en el control del peso del lodo puede generar problemas como colapsos (o comúnmente breakout) o fracturas [13]. Estos problemas son muy comunes y, además, son los mayores causantes 
de non productive time (NPT, es decir, tiempos no productivos), lo cual se asocia con pérdidas considerables de tiempo y dinero [14].

\section{Ventana de lodo}

La ventada de lodo es el rango de pesos de estos materiales, en los cuales las paredes del pozo tienen un grado de estabilidad óptimo [15]. La ventana de lodo tiene tres componentes principales: presión de la columna hidrostática de lodo $\left(\mathrm{P}_{\text {wc }}\right)$; presión de poro $\left(\mathrm{P}_{\mathrm{p}}\right)$ y la presión crítica de fractura $\left(\mathrm{P}_{\mathrm{wf}}\right)$. Los dos primeros representan el límite inferior, y lo ideal es no tener valores inferiores a estos, ya que algunas zonas de la perforación se diseñan con $\mathrm{P}_{\mathrm{wc}}$ para evitar derrumbes y otras con $\mathrm{P}_{\mathrm{p}}$ para evitar influjos. El límite superior de la ventana esta dado por $\mathrm{P}_{\mathrm{w} f}$, que controla eventos de pérdida de circulación.

Los modelos utilizados para el cálculo de las presiones críticas son objeto de profundización en este trabajo, ya que el cálculo de la $\mathrm{P}_{\mathrm{p}}$ tiene un abordaje totalmente diferente $\mathrm{y}$, por tanto, en las validaciones se asume que se describió durante la creación del modelo geomecánico.

\section{Desarrollo de modelos geomecánicos}

Para desarrollar los modelos geomecánicos, se llevó a cabo, primero, la revisión de literatura relacionada $\mathrm{y}$, segundo, la adaptación de planteamientos preexistentes. A partir de lo cual se generó la combinación de dos aspectos: (1) la concentración de los esfuerzos alrededor del pozo y (2) el criterio de falla. Este último describe la relación de falla entre la resistencia de la roca y los esfuerzos concentrados, estableciendo así los límites máximos antes de la falla de las rocas que, por consiguiente, genera inestabilidad en las paredes del pozo.

Enseguida, fueron propuestos los algoritmos para evaluar los modelos. Para ello, teniendo en cuenta que los algoritmos incluyen numerosos cálculos, se hizo necesario escribir un código en Matlab $^{\oplus}$, que evaluara dichos cálculos, para realizar las validaciones correspondientes.

Por último, se usaron modelos polares y cartesianos para el desarrollo de este trabajo. En el primero, varían todos los valores posibles de $\operatorname{Inc}_{\text {pozo }}\left(0-90^{\circ}\right)$ y Az $_{\text {pozo }}\left(0-360^{\circ}\right)$. Es decir, ninguno de los dos parámetros permanece constante. Por su parte, en el segundo modelo, se mantiene constante uno de los dos parámetros y se hacen variar los otros.

\section{Descripción del estado de esfuerzos alrededor del pozo}

El modelo del ingeniero alemán Ernst Kirsch [16] es el más usado para describir el tensor de esfuerzos alrededor de un pozo, concebido inicialmente para aplicaciones mineras y geotécnicas, pero puede usarse también de forma homóloga en pozos de petróleo. El modelo de Kirsch puede describir estados de esfuerzos totalmente anisotrópicos, pero bajo la condición de medio isotrópico y con deformación elástica lineal [17].

El punto más crítico cuando se evalúa dicho modelo es en $\mathrm{R}=\mathrm{rw}$, según la Amoco Corporation [10], para esa condición el modelo es el que muestra a continuación siguiendo las ecuaciones de Kirsch-ecuación la-e [16]:

$$
\begin{gathered}
\sigma_{r}^{\prime}=P_{w}-P_{p} \\
\sigma_{\theta}^{\prime}=\sigma_{x}^{\prime}+\sigma_{y}^{\prime}-2\left(\sigma_{x}^{\prime}-\sigma_{y}^{\prime}\right) \cos 2 \theta-4 \tau_{x y} \quad \sin 2 \theta-\left(P_{w}-P_{p}\right) \\
\sigma_{z z}^{\prime}=\sigma_{z}^{\prime}-2 v\left(\sigma_{x}^{\prime}-\sigma_{y}^{\prime}\right) \cos 2 \theta-4 \tau_{x y} \sin 2 \theta \\
\tau_{r \theta}=\tau_{r z}=0 \\
\tau_{z \theta}=2\left(-\tau_{x z} \cos \theta+\tau_{y z} \sin \theta\right)
\end{gathered}
$$


Donde:

$P_{w} \quad$ es la presión de la columna hidrostática de lodo (MPa).

$P_{p} \quad$ es la presión de poro (MPa).

$\sigma_{r, z z, \theta}$ es el esfuerzo normal efectivo radial, axial $\mathrm{y}$ tangencial $(\mathrm{MPa})$.

$\tau_{r \theta, r z, z \theta}$ es el esfuerzo cortante entre

$\sigma_{r}^{\prime}-\sigma_{\theta}{ }^{\prime}, \sigma_{r}^{\prime}-\sigma_{z}{ }^{\prime}, \sigma_{z}^{\prime}-\sigma_{\theta}^{\prime}(\mathrm{MPa})$.

$\sigma_{x, y, z}^{\prime}$ es el esfuerzo normal efectivo en la dirección $\mathrm{x}, \mathrm{y}, \mathrm{z}(\mathrm{MPa})$.

$\tau_{x y, x z, y z}$ es el esfuerzo cortante entre

$\sigma_{x}^{\prime}-\sigma_{y}^{\prime}, \sigma_{x}^{\prime}-\sigma_{z}^{\prime}, \sigma_{y}^{\prime}-\sigma_{z}^{\prime}(\mathrm{MPa})$.

$v$ es el módulo de Poisson (adimensional).

$\theta \quad$ es el ángulo sobre la circunferencia del pozo (sentido horario).

\section{Consideración para uso en pozos desviados}

A pesar de que las ecuaciones de Kirsch [16] fueron planteadas para $\sigma_{v}$ en el mismo eje del pozo y esfuerzos horizontales perpendiculares a él, es decir, un pozo vertical, por medio de transformaciones puede llevarse los tres esfuerzos principales a unos esfuerzos equivalentes ubicados como Kirsch los concibió. La transformación está en función tanto del azimut e inclinación del pozo como de la dirección del esfuerzo horizontal mayor, como muestra la ecuación 2 propuesta por Zoback [18], que está en términos de matrices y sus traspuestas.

$$
\begin{gathered}
{\left[\begin{array}{lllllll}
\sigma_{x} \tau_{x y} \tau_{x z} & \tau_{x y} & \sigma_{y} & \tau_{y z} & \tau_{x z} & \tau_{y z} \sigma_{z}
\end{array}\right]} \\
\quad=R_{1}\left[\begin{array}{llllllllll}
\sigma_{H} & 0 & 0 & 0 & \sigma_{h} & 0 & 0 & 0 & \sigma_{v}
\end{array}\right] R_{2}
\end{gathered}
$$

Donde:

$R_{1}, R_{2}=R_{b} R_{S}^{T}, R_{S} R_{b}^{T}$

$\sigma_{H}$ es el esfuerzo principal in situ horizontal mayor (MPa).

$\sigma_{h}$ es el esfuerzo principal in situ horizontal menor (MPa).

$\sigma_{v}$ es el esfuerzo principal in situ de sobrecarga $(\mathrm{MPa})$.
$R_{S}$ es la matriz de transformación respecto al Norte geográfico.

$R_{b}$ es la matriz de transformación respecto a la dirección del pozo.

Las matrices son expresadas como se muestra en la ecuación 3a-b.

$$
\begin{gathered}
R_{S}=\left[\cos \left(A z_{H}\right) \sin \left(A z_{H}\right) 0-\sin \left(A z_{H}\right)\right. \\
\left.\square \square \square \cos \left(A z_{H}\right) 0001\right] \\
R_{b}=[-a c-b c d b-a 0-a d b d c]
\end{gathered}
$$

Donde:

$a, b=\cos \left(A z_{\text {well }}\right), \sin \left(A z_{\text {well }}\right)$

$c, d=\cos \left(I n c_{w e l l}\right), \sin \left(I n c_{w e l l}\right)$

$A z_{\text {well }}$ es el azimut del pozo (en grados).

$I n c_{w e l l}$ es la inclinación del pozo (en grados).

$A z_{H} \quad$ es el azimut del esfuerzo horizontal mayor (en grados).

\section{Criterios de falla}

La deformación de una roca, al ser sometida a esfuerzos, depende de su elasticidad. Sin embargo, una vez excedidos los límites de resistencia la roca, esta fallará según el tipo de esfuerzo aplicado, es decir, si este es un esfuerzo de tensión o compresión [10].

Según Rahimi y Runar [19], para el caso de fallas tensionales, existe un único criterio; pero, para las fallas compresionales existe más variedad de criterios, ya que el fenómeno de dicha falla es más difícil de describir. En este caso, debe tenerse en cuenta el criterio de Mohr-Coulomb, el más usado y más fácil de evaluar. También es aplicable el criterio de Mogi-Coulomb y el de Lade modificado, que hacen mejores descripciones, ya que consideran los tres esfuerzos principales, contrario a Mohr-Coulomb que desprecia el intermedio.

Criterio de falla tensional o de fractura:

$$
\sigma_{3}^{\prime}=-T_{o}
$$

Criterio de falla compresional de Mohr-Coulomb:

$$
\sigma_{1}^{\prime}-q \sigma_{3}^{\prime}=U C S
$$




$$
q=\frac{1+\sin (A I F)}{1-\sin (A I F)}
$$

Criterio de falla compresional de Mogi-Coulomb:

$$
\begin{gathered}
\tau_{o c t}=a_{M C}+b_{M C} \sigma_{m} \\
\tau_{o c t}=\frac{1}{3} \sqrt{\left(\sigma_{1}-\sigma_{2}\right)^{2}+\left(\sigma_{1}-\sigma_{3}\right)^{2}+\left(\sigma_{2}-\sigma_{3}\right)^{2}} \\
\sigma_{m}=\frac{1}{2}\left(\sigma_{1}+\sigma_{3}\right)
\end{gathered}
$$

Criterio de falla compresional de Lade modificado

$$
\begin{gathered}
\frac{\left(I_{1}^{\prime}\right)^{3}}{I_{3}^{\prime}}=27+a_{L M} \\
I_{1}^{\prime}=\left(\sigma_{1}^{\prime}+b_{L M}\right)+\left(\sigma_{2}^{\prime}+b_{L M}\right)+\left(\sigma_{3}^{\prime}+b_{L M}\right) \\
I_{3}^{\prime}=\left(\sigma_{1}^{\prime}+b_{L M}\right)\left(\sigma_{2}^{\prime}+b_{L M}\right)\left(\sigma_{3}^{\prime}+b_{L M}\right) \\
a_{L M}=\frac{4 \tan ^{2}(A I F) \times(9-7 \sin (A I F))}{1-\sin (A I F)} \\
b_{L M}=\frac{U C S}{2 \sqrt{q}(A I F)}
\end{gathered}
$$

Donde:

$T_{0} \quad$ es la resistencia a la tensión (Mpa).

UCS es la resistencia a la compresión no confinada (Mpa).

AIF es el ángulo de fricción interna (en grados).

$\tau_{\text {oct }} \quad$ es el esfuerzo octaedral (Mpa).

$\sigma_{m} \quad$ es el esfuerzo medio (Mpa).

$q \quad$ es la constante del criterio de Mohr-Coulomb (adimensional).

$I_{1}^{\prime}, I_{3}^{\prime} \quad$ son los invariantes del criterio de Lade modificado.

$a_{M C}, a_{M C}$ son las constantes de criterio de Mogi-Coulomb.
$a_{L M}, a_{L M}$ son las constantes de criterio de Lade modificado.

\section{Esfuerzos principales alrededor del pozo}

En un pozo orientado en la misma dirección de esfuerzos in situ (pozo vertical o pozo horizontal en el mismo azimut de esfuerzos horizontales), los esfuerzos principales de la pared del pozo coinciden con $\sigma_{\mathrm{r}}, \sigma_{z z}$ y $\sigma_{\theta}$. Pero, en un pozo desviado, esto no se cumple, y deben tenerse en cuenta los esfuerzos cortantes. Por lo que es necesario generalizar el estado de esfuerzos principales de la pared del pozo. Según Mitchell et al [20], estos esfuerzos se definen de la siguiente manera:

$$
\begin{gathered}
\sigma_{1,3}^{\prime}=\sigma_{r} \quad(8 \mathrm{a}) \\
\sigma_{1,2,3}^{\prime}=\frac{1}{2}\left(\sigma_{\theta}^{\prime}+\sigma_{z z}^{\prime}\right) \pm \frac{1}{2} \sqrt{\left(\sigma_{\theta}^{\prime}-\sigma_{z z}^{\prime}\right)^{2}+4 \tau_{\theta z}^{2}}
\end{gathered}
$$

Donde $\sigma_{1,2,3}^{\prime}$ es el esfuerzo normal efectivo principal mayor, intermedio y menor.

Según las figuras 2 y 4 , las fallas tensionales están asociadas con altos valores del peso de lodo, contrario a las compresionales. Por ello, en el caso de fallas tensionales, $\sigma_{1}=\sigma_{r}$, y en compresionales, $\sigma_{3}=\sigma_{r}$; los esfuerzos principales restantes se asignan por defecto. Este concepto es importante al momento de combinar el modelo de Kirsch [17] con los esfuerzos principales.

\section{Presiones críticas}

Son las presiones que definen los límites de las fallas alrededor del pozo; de ello deriva la aplicación de todos los modelos planteados, toda vez que, con base en ellas, se construye la ventana de lodo. Al reemplazar los esfuerzos que describe el modelo de Kirsch [17] (ecuación 1) en los esfuerzos principales (ecuación 8), y luego estos últimos en los criterios de falla (ecuaciones 4-7), se obtienen las ecuaciones de las presiones críticas. 
Presión crítica de fractura:

$$
\begin{aligned}
P_{w f}=\left\{\sigma_{x}^{\prime}+\right. & \sigma_{y}^{\prime}-2\left(\sigma_{x}^{\prime}-\sigma_{y}^{\prime}\right) \cos (2 \theta)-4 \tau_{x y} \sin (2 \theta)-\frac{\tau_{x y}^{2}}{\sigma_{z z}^{\prime}+T_{o}} \\
& \left.+P_{p}+T_{o}\right\}
\end{aligned}
$$

Presión crítica de colapso (Mohr-Coulomb):

$$
f\left(P_{w c}\right)=\frac{1}{2}\left[A-P_{w c}\right]+\frac{1}{2} \sqrt{\left[B-P_{w c}\right]^{2}+C}-U C S-q\left(P_{w c}-P_{p}\right)=0
$$

Presión crítica de colapso (Mogi-Coulomb):

$$
\begin{gathered}
f\left(P_{w c}\right)=\frac{1}{3}\left\{\left[\sqrt{\left[B-P_{w c}\right]^{2}+C}\right]^{2}\right. \\
+\left[\frac{1}{2}\left[A-P_{w c}\right]-\frac{1}{2}\left[\overline{\left[B-P_{w c}\right]^{2}+C}-P_{w c}+P_{p}\right]^{2}\right. \\
\left.+\left[P_{w c}-P_{p}-\frac{1}{2}\left[A-P_{w c}\right]-\sqrt{\left[B-P_{w c}\right]^{2}+C}\right]^{2}\right\}^{\frac{1}{2}} \\
-a_{M C}-b_{M C}\left\{\frac { 1 } { 2 } \left[\frac{1}{2}\left[A-P_{w c}\right]-\frac{1}{2} \sqrt{\left[B-P_{w c}\right]^{2}+C}\right.\right. \\
\left.+P_{w c}-P_{p}\right]\{=0
\end{gathered}
$$

Presión crítica de colapso (Lade modificado)

$$
\begin{gathered}
f\left(P_{w c}\right)=-27-a_{L M} \\
+\left\{\frac{\left(A-P_{w c}-P_{p}+3 b_{L M}\right)^{3}}{\frac{1}{2}\left[A-P_{w c}\right]+\frac{1}{2} \sqrt{\left[B-P_{w c}\right]^{2}+C}+b_{L M}}\right\} \\
\times\left\{\frac{1}{\frac{1}{2}\left[A-P_{w c}\right]-\frac{1}{2} \sqrt{\left[B-P_{w c}\right]^{2}+C}+b_{L M}}\right\} \\
\times\left\{\frac{1}{P_{w c}-P_{p}+b_{L M}}\right\}=0
\end{gathered}
$$

Donde:

$A=\sigma_{x}^{\prime}+\sigma_{y}^{\prime}-2\left(\sigma_{x}^{\prime}-\sigma_{y}^{\prime}\right) \cos 2 \theta-P_{p}+\sigma_{z z}^{\prime}$

$B=A-2 \sigma_{z z}^{\prime}$

$C=4 \tau_{z \theta}^{2}$

$P_{w f}$ es la presión crítica de fractura.

$P_{w c}$ es la presión crítica de colapso. 


\section{Resultados y discusión}

\section{Condiciones de aplicación}

Según el modelo de Kirsch [17], la descripción es para medios con deformación elástica lineal. Además, usando la trasformación de la ecuación 2, según Zoback [19], es posible que pueda aplicarse a pozos con cualquier desviación.

\section{Algoritmos para evaluar los modelos}

Para evaluar las ecuaciones planteadas y programarlas en $\mathrm{Matlab}^{\odot}$ se plantearon las consideraciones que se describen a continuación.

Presión crítica de fractura. El procedimiento consiste, principalmente, en calcular $\mathrm{P}_{\mathrm{wf}}$ en cada punto del pozo. Es decir, para cada $\theta$ y, en el caso de las fallas tensionales, el punto más crítico es donde $\mathrm{P}_{\mathrm{wf}}$ sea menor, ya que se trata de un límite inferior, pues por encima de este empiezan a formarse las fracturas, de modo que se propone el algoritmo de la figura 1.

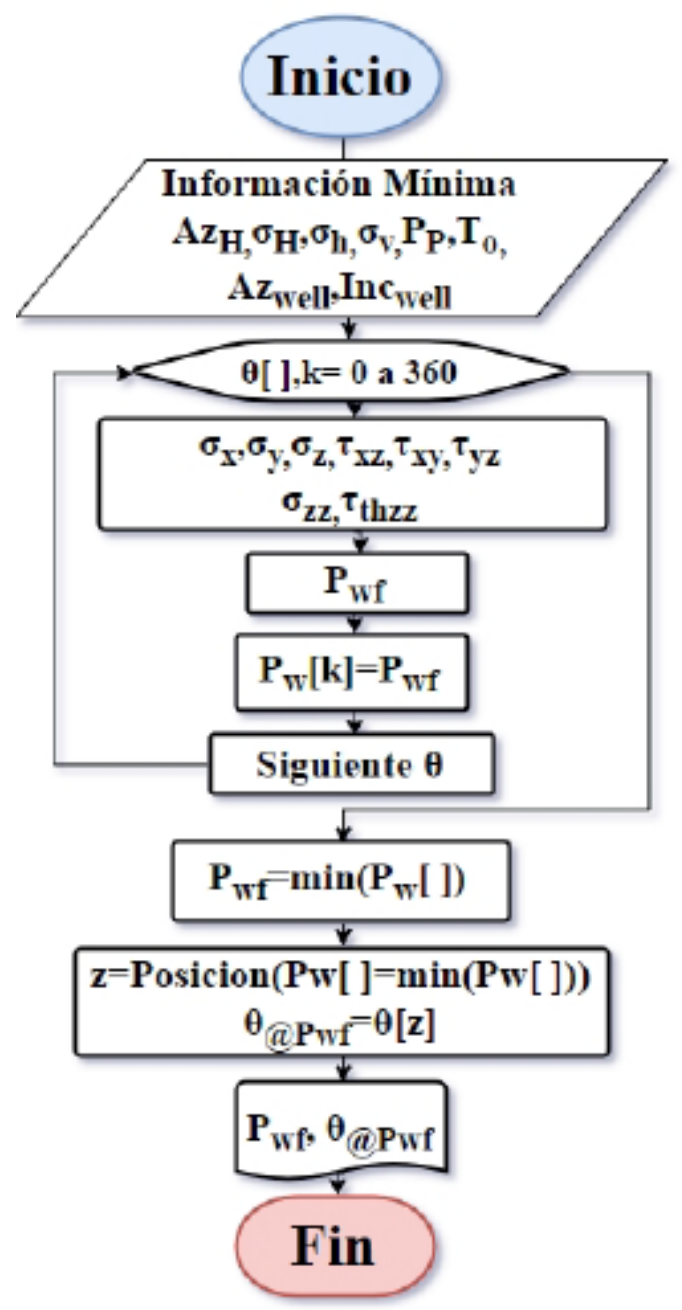

Figura 1. Algoritmo para evaluar la presión crítica de fractura.

Fuente: elaboración propia. 
Presión crítica de colapso. El procedimiento para calcular $\mathrm{P}_{\mathrm{wc}}$, con respecto a $\mathrm{P}_{\mathrm{w} f}$, se diferencia en que el punto más crítico ya no es la menor presión sino la mayor; además de que ese valor depende también de la tolerancia definida al breakout $\left(\mathrm{W}_{\mathrm{br}}\right)$ que se tenga definido. Esto último se debe a que los breakout, a diferencia de las fracturas, tienen un ancho, evidentemente cuanto menor sea dicha tolerancia más estable es el pozo. Sin embargo, la práctica ha demostrado que puede manejarse una tolerancia y conservar la condición de estabilidad.

La segunda condición importante es la necesidad de aplicar métodos numéricos. Luego de hacer evaluaciones se concluyó que la iteración simple no funcionaba bien para todos los modelos, por lo que se utilizó un método más elaborado: NewtonRaphson mostró los mejores resultados y velocidad de convergencia, con el cual se propuso el siguiente algoritmo (figura 2).



Fin

Figura 2. Algoritmo para evaluar la presión crítica de colapso.

Fuente: elaboración propia. 
Al usar el método de Newton-Raphson se presentó una consideración adicional: debe hallarse la derivada de cada expresión propuesta. Para el caso de Mohr- Coulomb, no hay mayores inconvenientes para derivar. Sin embargo, para los demás criterios las ecuaciones son muy complejas de derivar. Para solucionar esta dificultad se usó la función $\operatorname{diff}[f(X), X]$ de Matlab $^{\odot}$.

\section{Validación de los modelos}

Los datos de entrada para evaluar las presiones críticas son, principalmente, el estado de esfuerzos in situ; las propiedades de resistencia de la roca; y la presión de poro. En conjunto, estas variables se conocen como modelo geomecánico. Las evaluaciones se hicieron con un programa escrito en $\mathrm{Matlab}^{\odot}$, en el que los códigos principales se basan en los algoritmos mostrados en las figuras 1 y 2 .

\section{Modelo de presión crítica de fractura}

Para desarrollar un ejercicio práctico, se tomaron como datos de entrada:

$$
\begin{aligned}
\mathrm{T}_{0} & =0 \mathrm{MPa} \\
\alpha_{\mathrm{H}} & =70^{\circ} \\
\sigma_{\mathrm{v}} & =70 \mathrm{MPa} \\
\sigma_{\mathrm{H}} & =55 \mathrm{MPa} \\
\sigma_{\mathrm{h}} & =45 \mathrm{MPa} \\
\mathrm{P}_{\mathrm{p}} & =32 \mathrm{MPa}
\end{aligned}
$$

Con el algoritmo propuesto, se obtuvo el diagrama de ventana de lodo tensional polar en los cuales los círculos concéntricos son Inc $\mathrm{pozo}_{\text {, }}$ ubicados en el centro un pozo vertical y en la periferia un pozo horizontal. Adicionalmente, las líneas radiales son $\mathrm{Az}_{\text {pozo }}$ (figura 3).

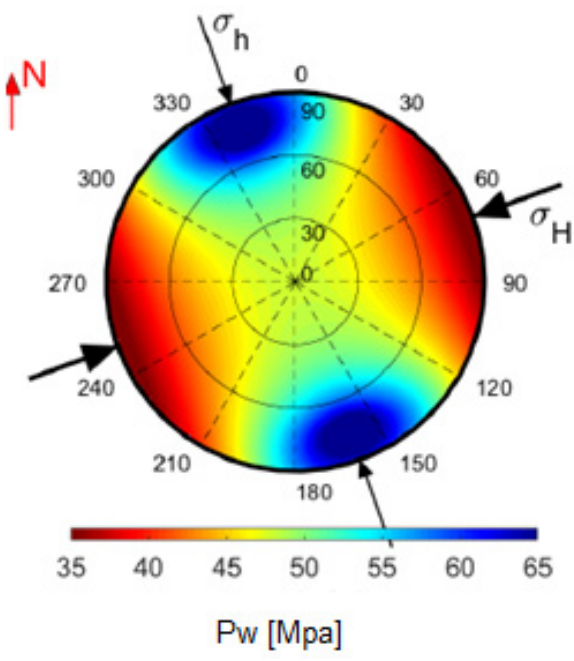

Figura 3. Ventana de lodo tensional polar.

Fuente: elaboración propia.

\section{Modelos de presión crítica de colapso}

Para desarrollar un ejercicio práctico, se tomaron como datos de entrada:

$$
\begin{aligned}
& \mathrm{UCS}=17,15 \mathrm{MPa} \\
& \mathrm{AIF}=40^{\circ} \\
& \nu=0,31
\end{aligned}
$$

$$
\begin{aligned}
& \alpha_{\mathrm{H}}=0^{\circ} \\
& \sigma_{\mathrm{V}}=80 \mathrm{MPa} \\
& \sigma_{\mathrm{H}}=60 \mathrm{MPa} \\
& \sigma_{\mathrm{h}}=58 \mathrm{MPa} \\
& \mathrm{P}_{\mathrm{p}}=34,5 \mathrm{MPa}
\end{aligned}
$$

Utilizando el algoritmo propuesto, se obtuvo el modelo de presión crítica de colapso. Los 
diagramas, en este caso, son cartesianos. En ellos se toma como valor fijo $A z_{\text {pozo }}=90^{\circ}$, donde, en el eje $\mathrm{X}$, se varía el $\mathrm{Inc}_{\mathrm{pozo}}$; $\mathrm{y}$, en el eje $\mathrm{Y}$, se obtiene el overbalanced $\left(\mathrm{P}_{\mathrm{w}}-\mathrm{P}_{\mathrm{P}}\right)$ necesario según el modelo aplicado (figura 4).

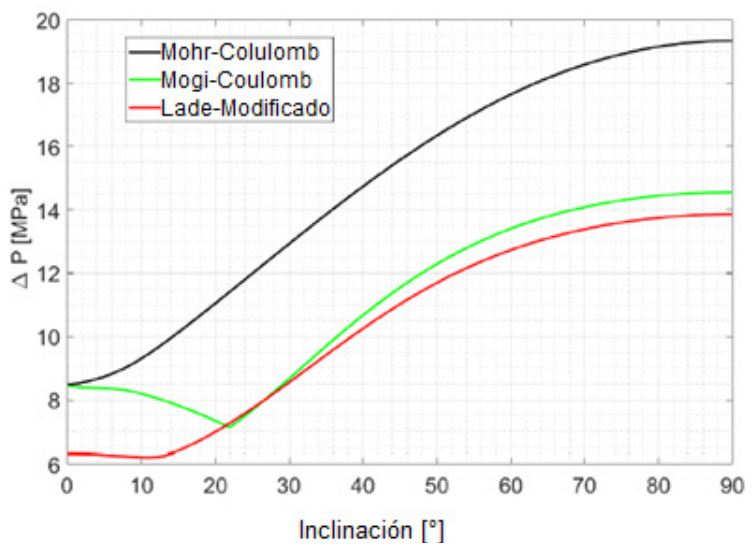

Figura 4. Ventana de lodo compresional cartesiana. Fuente: elaboración propia.

Pueden obtenerse interpretaciones en términos de estabilidad, que brindan los dos grupos de gráficas; además, las comparaciones respectivas entre lo que obtuvo el autor de referencia y el modelo propuesto aquí.

Para el caso del modelo tensional, con respecto a la presión crítica tensional observada, las regiones óptimas (donde $\mathrm{P}_{\mathrm{wf}}$ es mayor) coinciden con $\sigma_{\mathrm{h}}$; mientras que las menos recomendadas están en la dirección perpendicular. En cuanto a los análisis de estabilidad, puede afirmarse que las condiciones óptimas se encuentran para $A z_{\text {pozo }}$ entre

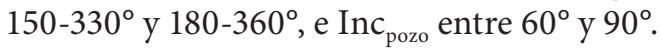

Por otro lado, para el modelo compresional, en la descripción de la presión crítica de colapso, se observa que el overbalanced que describe MohrCoulomb es de los mayores; mientras que en Lade modificado se encuentran los menores. Ello se explica en que el criterio e Mohr-Coulomb desprecia el efecto del esfuerzo principal intermedio, lo que hace que la roca aparentemente esté sometida a menor compresión, por lo que tendrá menos resistencia y tenderá a describir que se requieren mayores valores de $\mathrm{P}_{\mathrm{wc}}$ para lograr la estabilidad. Es decir, el criterio es más conservador que los otros.

En cuanto a los análisis de estabilidad, este tipo de gráficas solo permite evaluar pozos verticales o paralelos a uno de los horizontales, en este caso $\sigma_{\mathrm{h}}$ (porque $\mathrm{Az}_{\mathrm{pozo}}=90^{\circ}$ ). Puede verse que el menor valor del overbalanced y, por tanto, la dirección más estable, se encuentra para $\mathrm{Inc}_{\text {pozo }}$ en $0-20^{\circ}$ aproximadamente. Esto puede explicarse, principalmente, por que la diferencia de los valores de los esfuerzos horizontales es muy pequeña; esto hace que los pozos de poca inclinación tengan una distribución más uniforme de esfuerzos en comparación a los pozos altamente desviados.

\section{Conclusiones}

Se desarrollaron dos modelos analíticos y sus algoritmos de evaluación, que puedan ser usados en el cálculo de las presiones críticas de la ventana de lodo.

La presión crítica de fractura se calculó teniendo en cuenta el criterio recomendado por la literatura, es decir, el criterio de Mohr-Coulomb, caracterizado por ser el más elemental y fácil de evaluar. Sin embargo, debe considerarse que los criterios de Mogi-Coulomb y Lade modificado hacen descripciones más acertadas.

En modelos tensionales, con respecto a la presión crítica tensional, las regiones óptimas (donde $\mathrm{P}_{\mathrm{wf}}$ es mayor) coinciden con $\sigma_{\mathrm{h}}$; y los análisis de estabilidad se encuentran para $A z_{\text {pozo }}$ entre $150-330^{\circ}$ y $180-360^{\circ}$, e Inc $c_{\text {pozo }}$ entre $60^{\circ}$ y $90^{\circ}$.

Por otro lado, los menores valores del overbalanced concuerdan con la dirección más estable que se encuentra para $\operatorname{Inc}_{\text {pozo }}$ entre 0 y $20^{\circ}$, aproximadamente. Ello hace que los pozos de poca inclinación tengan una distribución más uniforme de esfuerzos, en comparación con los pozos altamente desviados.

Se destacó la importancia del diseño de la ventana de lodo, ya que la estabilidad del pozo es uno de los mayores causantes de pérdidas de tiempo y recursos económicos, durante operaciones de perforación. 


\section{Referencias}

[1] H. Mora-Páez y C. M. Jaramillo, “Aproximación a la construcción de cartografía social a través de la geomática", Ventana Informática, vol. 11, pp. 129-146, 2018.

[2] O. Heidbach et al., "The World Stress Map Database Release 2016: Crustal Stress Pattern Across Scales”, Tectonophysics, vol. 744, pp. 484-498, 2018. Doi: https://doi. org/10.1016/j.tecto.2018.07.007

[3] H, Soroush, "Discover a Career in Geomechanics." The Way Ahead, vol. 9, n. ${ }^{\circ}$ 3, pp. 15, 2013. Doi: https://doi. org/10.2118/0313-015-TWA

[4] M. A. Addis, "The Geology of Geomechanics: Petroleum Geomechanical Engineering in Field Development Planning”, Geological Society, London, Special Publications, vol. 458, n. ${ }^{\circ}$ 1, pp. 7-29, 2017. Doi: https:// doi.org/10.1144/SP458.7

[5] G. Osorio y C. F. Lopez, "Geomechanical Factors Affecting the Hydraulic Fracturing Performance in a Geomechanically Complex, Tectonically Active Area in Colombia", en Latin American and Caribbean Petroleum Engineering Conference, Cartagena de Indias, Colombia, 2009. Doi: https://doi.org/10.2118/122315-MS

[6] F. Fernández-Ibáñez et al., "3D Geomechanical Modeling for the Apiay and Suria Oil Fields (Llanos Orientales Basin, Colombia): Insights on the Stability of Reservoir-Bounding Faults", en SPE Latin American and Caribbean Petroleum Engineering Conference, Lima, Perú, 2010. Doi: https://doi.org/10.2118/138869-MS

[7] C. Guerra, K. Fischer y A. Henk, "Stress Prediction Using 1D and 3D Geomechanical Models of a Tight Gas Reservoir -A Case Study from the Lower Magdalena Valley Basin, Colombia”, Geomechanics for Energy and the Environment, vol. 19, p. 100113, 2019. https://doi.org/10.1016/j.gete.2019.01.002

[8] L. A. Medina y A. N. Tutuncu, "3-D Geomechanical Modeling for Field Development of a Colombian Shale Play," en Unconventional Resources Technology Conference (URTec) of Society of Exploration Geophysicists, Denver, Colorado, 2019, pp. 3897-3916. Doi: https://doi.org/10.15530/urtec-2019-467
[9] O. Carvajal y C. Sierra, "Risk Mitigation in Overpressured Wells Through Geomechanical Approach", en SPE Latin American and Caribbean Petroleum Engineering Conference, Virtual, 2020. Doi: https://doi. org/10.2118/199141-MS

[10] Amoco Corporation -Amoco, "Wellbore Stability Drilling Handbook”. EE. uU., Amoco Corporation, 1997.

[11] R. F. Mitchell y S. Z. Miska, "Fundamentals of Drilling Engineering", Houston [Tx]: Country of publication: Society of Petroleum Engineers, 2011.

[12] X. Chen, C. P. Tan y C. M. Haberfield, “A Comprehensive, Practical Approach for Wellbore Instability Management", SPE Drilling \& Completion, vol. 17, n. ${ }^{\circ}$ 04, pp. 224-236, 2002. Doi: https://doi.org/10.2118/80146-PA

[13] S. Li, J. George y C. Purdy, "Pore-pressure and Wellbore-Stability Prediction to Increase Drilling Efficiency", Journal of Petroleum Technology, vol. 64, n. ${ }^{\circ}$ 02, pp. 98101, 2012. Doi: https://doi.org/10.2118/144717-JPT

[14] C. Anyanwu et al., "Analysis of Wellbore Stability in Multilateral Well Design and Construction", en SPE Nigeria Annual International Conference and Exhibition, Lagos, Nigeria, 2013. Doi: https:/doi.org/10.2118/167546-MS

[15] S. Wessling et al., "Automation in Wellbore Stability Workflows", en SPE Intelligent Energy International, Utrecht, The Netherlands, March, 2012. Doi: https:// doi.org/10.2118/149766-MS

[16] E. G. Kirsch, Die Fundamentalgleichungen der Theorie der Elasticität fester Körper, hergeleitet aus der Betrachtung eines Systems von Punkten, welche durch elatische Streben verbunden sind. Berlin, Heidelberg: Elsevier, 1868.

[17] B. Aadnoy et al., "Advanced Drilling and Well Technology", Houston [Tx]: Society of Petroleum Engineers, 2009, pp. 301-440.

[18] M. D. Zoback, "Reservoir geomechanics”, Cambridge [UK]: Cambridge University Press, 2010.

[19] R. Rahimi y N. Runar, "What Difference Does Selection of Failure Criteria Make in Wellbore Stability Analysis?"; en 48th US Rock Mechanics/Geomechanics Symposium, Minneapolis, Minnesota, 2014.

[20] R. F. Mitchell, K. Ravi y Pgs. H, "Volume II-Drilling Engineering", Richardson [Tx]: Society of Petroleum Engineers, 2006. 